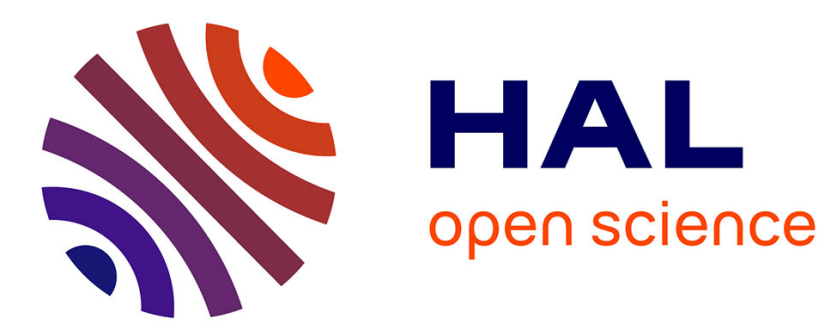

\title{
Une action collective sous contrainte européenne? Le cas des viticulteurs "précarisés" dans le département de l'Aude
}

Stéphanie Dechezelles, Antoine Roger

\section{To cite this version:}

Stéphanie Dechezelles, Antoine Roger. Une action collective sous contrainte européenne? Le cas des viticulteurs "précarisés" dans le département de l'Aude. Raison Présente, 2009, Action collective et exclusion sociale, 170, pp.23-37. 10.3406/raipr.2009.4157 . hal-01816072

\section{HAL Id: hal-01816072 \\ https://hal.science/hal-01816072}

Submitted on 14 Jun 2018

HAL is a multi-disciplinary open access archive for the deposit and dissemination of scientific research documents, whether they are published or not. The documents may come from teaching and research institutions in France or abroad, or from public or private research centers.
L'archive ouverte pluridisciplinaire HAL, est destinée au dépôt et à la diffusion de documents scientifiques de niveau recherche, publiés ou non, émanant des établissements d'enseignement et de recherche français ou étrangers, des laboratoires publics ou privés. 


\title{
Une action collective sous contrainte européenne?
}

\section{Le cas des viticulteurs « précarisés » dans le département de l'Aude}

\author{
Stéphanie DECHEZELLES \\ (Université de Bordeaux, Sciences Po Bordeaux, SPIRIT UMR 5116) \\ Antoine ROGER \\ (Université de Bordeaux, Sciences Po Bordeaux, SPIRIT UMR 5116)
}

\begin{abstract}
Article à paraître dans Raison présente [article accepté, parution prévue pour juin 2009]
\end{abstract}
Nombre d'agriculteurs français se trouvent aujourd'hui pris dans une dynamique de précarisation, c'est-à-dire de relégation dans « un espace de la fragilité ou de la vulnérabilité sociale et économique qui est marqué par un rapport incertain à l'avenir ». Le terme de précarité recouvre quatre dimensions ${ }^{1}$ : une «insécurité matérielle ou économique»; une " insécurité statutaire », qui procède d'une « impossibilité de se protéger contre les aléas de l'existence »; une faible marge de manœuvre, qui restreint les choix à disposition des individus; une "indignité sociale», enfin, sublimée sous certaines conditions et dans certaines conjonctures par une "construction sociale des identités précaires » et par une «gestion militante» (Boumaza et Pierru, p. 11-14). La première dimension est aisément observable dans des secteurs de l'agriculture française où les bas revenus sont très nombreux ; il apparaît du reste que la consommation globale des agriculteurs est inférieure de $20 \%$ en moyenne à celle de l'ensemble des actifs non agricoles. La deuxième dimension peut également être caractérisée : beaucoup d'agriculteurs vivent à distance des structures d'aide à l'emploi et sont privés de l'accès aux soins (Jégouzo, Brangeon, \& Roze, 1998). Les obstacles (naturels, techniques et financiers) qui empêchent de développer de nouvelles activités rémunératrices donnent à voir la troisième dimension. La quatrième dimension est plus significative encore : il n'existe guère à l'heure actuelle de mouvement structuré qui prétende défendre les agriculteurs en difficulté. La publicisation et la politisation de la cause restent très faibles : tout se passe comme si les difficultés socioéconomiques rencontrées dans le secteur étaient prises dans un mécanisme d'invisibilisation sociale. Cette particularité attire l'attention sur l'impérieuse nécessité pour toute entreprise de mobilisation de construire et de « faire parler» la cause (Collovald \& Gaïti, 1991). La compréhension des procédés qui servent à mettre un problème en forme, aussi bien qu'en mots, compte parmi les apports stabilisés des travaux sur l'action collective et les mouvements sociaux. Les mobilisations agricoles ne font pas exception : une revendication d'agriculteurs ne peut venir au jour que dans la mesure où elle est travaillée, élaborée et labellisée (Maresca, 1983). La question se pose alors de savoir pourquoi la situation des agriculteurs français les plus en difficulté ne fait aujourd'hui l'objet d'aucune prise en charge spécifique.

Pour apporter quelques éléments de réponse - nécessairement partiels et provisoires - nous nous concentrerons sur le cas particulier de la viticulture. Ce secteur d'activité offre un poste d'observation pertinent dans la mesure où il a prêté par le passé à de fortes mobilisations à l'échelle nationale (Guillemin, 1990; Mann, 1997), en parfait contraste avec l'atonie

\footnotetext{
${ }^{1}$ La notion de précarité est investie par des « discours hybrides » (médiatiques, politiques et académiques) dont procèdent d'importants «enjeux de légitimation»: «la conceptualisation s'appuie sur une représentation légitime de la cohésion sociale, qu'elle contribue aussi à promouvoir » (Bresson, 2007, p. 13). Raisonner en termes de "précarisation» ou de «mise en précarité » permet de contrôler un tant soit peu le flottement terminologique: il ne s'agit pas de définir une catégorie substantielle mais de caractériser les éléments qui contribuent à une classification et à une auto-qualification.
} 
aujourd'hui constatée. Occupant le cœur de cible des réformes portées par l'Union Européenne (UE), il est engagé dans un vaste processus de restructuration qui doit conduire à une élimination économique des producteurs les plus modestes. Le vin européen peinant à s'affirmer sur les marchés internationaux en raison de la concurrence exercée par les vins dits $\mathrm{du}$ « Nouveau Monde $»^{2}$, la Commission européenne s'attache à réformer l'Organisation Commune de Marché (OCM) qui régule la production et le négoce à l'échelle de l'UE. Dans un document publié le 22 juin 2006 sous le titre Vers un secteur vinicole européen durable, Mariann Fischer Boel (Commissaire européenne à l'Agriculture et au développement rural) annonce des mesures qui doivent être adoptées en 2008. L'objectif affiché est de faire évoluer « le modèle viticole européen, majoritairement artisanal et trop dépendant de subventions ", vers « un modèle plus industriel et plus compétitif ». La Commission entend ainsi réduire la production de vin dans l'UE, en subventionnant durant cinq ans l'arrachage de 175.000 hectares de vignes ${ }^{3}$ et en mettant un terme au système de distillation de crise qui a jusqu'alors permis de transformer les stocks de vins invendus en alcools de bouche pour l'industrie des spiritueux. Elle propose aux «producteurs non compétitifs » de se "retirer du secteur » dans des « conditions financières satisfaisantes ». Le $1^{\text {er }}$ janvier 2014, une libéralisation complète des droits de plantation doit par ailleurs être engagée : les «producteurs compétitifs 》 restés sur le marché pourront alors accroître leur production en fonction de leur capacité à exporter.

La réforme ainsi engagée condamne l'activité même de nombreux viticulteurs. Pour prendre la mesure de ses effets, nous proposons de porter un éclairage particulier sur le département français de l'Aude $^{4}$. Ce terrain d'étude est particulièrement visé par la restructuration annoncée. Longtemps cantonné à la production d'un vin de consommation courante (le « vin de table »), il a connu dans les années 1980 une " révolution qualité » : en appui sur des fonds européens, une politique de replantage a permis de développer un "encépagement noble » (merlot, cabernet, syrah...) en faisant le choix d'une baisse des rendements. Les Appellations d'Origine Contrôlée (AOC) se sont dès lors multipliées. Elles sont aujourd'hui directement frappées par la concurrence. Dans une gamme identique, les vins du « Nouveau Monde » sont en effet moins chers, plus clairement identifiables par les consommateurs (l'étiquetage en « vin de cépage » offre une meilleure lisibilité que le dédale des appellations) et présentent une qualité constante (des techniques œnologiques peu restrictives permettent de palier les effets des aléas climatiques). En conséquence, nombre de producteurs audois peinent à écouler leur production à des prix qui permettraient d'obtenir un retour sur investissements. Selon les chiffres fournis par la Chambre d'agriculture de l'Aude, le département compte 7562 viticulteurs. 119 d'entre eux percevaient le Revenu Minimum d'Insertion (RMI) en 2005 contre 435 en 2006. Près de 3000 sont aujourd'hui proches du dépôt de bilan ${ }^{5}$. Les pertes moyennes sont estimées à 1000 euros par an et par hectare depuis 2004. La baisse des revenus atteint $40 \%$ par an en moyenne, qui n'est pas ou très peu compensée par des activités parallèles (tourisme, autres productions...).

Les exploitants établissent le plus souvent un lien direct entre leurs difficultés matérielles et la politique viticole de l'UE. Les entretiens réalisés laissent poindre des incriminations claires, dont quelques exemples peuvent être donnés :

\footnotetext{
${ }^{2}$ Les vignobles de Californie, d'Australie, du Chili, etc. sont plus rentables car cultivés sur de plus vastes étendues. A titre de comparaison, la surface moyenne d'une exploitation est de 100 hectares en Australie et de 8 hectares en France.

${ }^{3}$ Dans un premier temps, l'arrachage de 400.000 hectares est annoncé. En réponse aux salves de critiques, le chiffre est ensuite revu à la baisse.

${ }^{4}$ Notre propos est appuyé sur une analyse documentaire (documents officiels, publications locales, tracts...) et sur des entretiens réalisés en 2007 avec des viticulteurs " précarisés 》 des Corbières et du Minervois.

${ }^{5}$ Chaque exploitation viticole assure 3 à 5 emplois induits.
} 
« On tue des hommes, des familles (...) C'est la politique de l'Europe qui tue les gens. On est en train de mettre en place des trucs atroces » (viticulteur des Corbières)

« Ce que font les dirigeants européens qui regardent ça de très haut, de très loin, Mme Boel et les autres, c'est de dire : 'Bon, on comprend que jamais ils ne seront performants, donc on va les aider à partir'. Nous on fait partie de ceux qui souffrent de ça...» (viticulteur du Minervois)

« Voila ce que dit Mariann, hein! 'Vous n’êtes pas compétitifs, barrez vous ! Voilà les sous ! Payez vos emprunts!'. Moi j'ai 55 ans, si je décide de rien faire et de me barrer, j'arrache tout, je vais rentrer un capital, avec ce capital je vais rembourser le Crédit agricole, et je bouffe quoi après ? » (viticulteur du Minervois)

En dépit de cette capacité manifeste d'imputation, aucune mobilisation significative contre les choix de la Commission n'est enregistrée. Des responsables sont bien identifiés de façon plus ou moins nominative («la politique de l'Europe», «les dirigeants européens », la Commissaire à l'agriculture et au développement rural), mais les protestations restent faiblement articulées et aucun porte-parole ne vient formuler des revendications audibles. Déroutante au premier abord, cette configuration peut être mise sur le compte d'une structuration ambivalente : en même temps qu'elle fournit des «polarisateurs» de mobilisation, l'Union européenne provoque de façon plus discrète des évolutions qui grippent indirectement le processus de construction de la cause. Sans participer d'un projet concerté, des aménagements institutionnels sont réalisés qui font obstacle à la mise en forme et à l'expression de revendications collectives dans certains secteurs de production. Les viticulteurs en difficulté se voient proposer des solutions individuelles et ne disposent d'aucun support pour engager un combat unitaire. Ces obstacles pourraient encore être surmontés si des organisations représentatives s'attachaient à délimiter précisément les contours d'un groupe dont elles se proclameraient les porte-parole; à l'inverse, la politique viticole européenne alimente des conflits multiples et enchevêtrés qui compliquent singulièrement l'exercice d'objectivation et font obstacle à toute mobilisation.

\section{Une individualisation des difficultés vécues}

En imitation des schémas diffusés par la Commission européenne, les dispositifs nationaux mis en place pour traiter les difficultés matérielles (parfois anciennes) des viticulteurs invitent chacun à formuler une demande d'assistance individuelle; la réception de discours qui tendraient à généraliser le propos et à l'inscrire dans un cadre collectif est alors compromise.

Des travaux empiriques révèlent que la forme individualisante du traitement social fait obstacle à la construction d'une cause portée collectivement. Comme le montre Serge Ebersold, l'accent est placé sur la responsabilité individuelle des demandeurs d'emploi, ce qui empêche la construction d'un problème collectif (Ebersold, 2001).

La politique viticole européenne aménage progressivement un régime du même ordre. En 1962, les six Etats fondateurs de la Communauté économique européenne mettent en place l'OCM dans le but d'harmoniser les principes qui encadrent la production et le commerce du vin. Chacun conserve néanmoins une grande marge de manœuvre pour la classification des produits, la fixation des droits de plantation et la définition des règles œnologiques. En réponse à la crise des années 1970, les gouvernements nationaux s'accordent pour donner une orientation plus dirigiste au dispositif : 1'OCM interdit alors la plantation de nouvelles vignes et permet une distillation des excédents avantageuse pour les producteurs. Les mesures 
conservent un caractère global et ne s'appliquent pas sur une base individuelle. Pour chaque campagne, les distillateurs se voient imposer un prix minimal d'achat pour chaque catégorie de vin ; ils reçoivent des subventions compensatoires, prélevées sur les fonds communautaires (Smith, de Maillard, Costa, 2007). En 1980, ce dispositif est remis en cause : la Commission européenne voit dans le versement indifférencié de subventions automatiques un encouragement à la passivité. Elle préconise alors de récompenser les initiatives et les efforts individuels. Un régime d'aides à la restructuration et au réencépagement des vignobles est mis en place. Il vise particulièrement le Languedoc-Roussillon, accusé de privilégier la quantité au détriment de la qualité et de produire un vin de table inadapté à l'évolution des pratiques de consommation. Les viticulteurs sont invités à élaborer des dossiers pour obtenir des subventions. Qu'elles émanent d'exploitations particulières ou d'un rassemblement de producteurs, les demandes sont formulées isolément et étudiées au cas par cas. Au cours des entretiens personnalisés qui servent à les préparer, les agents de la Commission mettent l'accent sur la nécessité de prendre des risques commerciaux et de développer une stratégie d'entreprise. La restitution d'une expérience individuelle permet de saisir la portée de cette démarche injonctive.

\footnotetext{
«Quand je suis devenu directeur d'un groupement, mon intention était, sur 8 villages, 8 caves coopératives et à peu près 150.000 hectos de production, de tirer par le haut mais de faire en sorte que les gens soient impliqués par l'acte commercial. Je sentais bien que sans eux, on ne pouvait vendre que par le prix. Comme directeur d'un groupement de producteurs, si vous avez devant vous 150.000 hectos de merde, l'Europe est là pour vous dire, mettez donc en place par les schémas directeurs un autre type de viticulture qualitative et on va vous aider à monter les structures de mise en marché.» (viticulteur du Minervois).
}

En 1993, le système d'aide à la reconversion est réformé. Financées par le Fonds européen d'orientation et de garantie agricole (FEOGA), les subventions sont désormais gérées par les Etats membres. La Commission élabore des recommandations et met un guide à la disposition des autorités nationales: des critères sont fournis pour aider à «faire le tri » entre les exploitants dont il convient de soutenir l'activité et ceux qui sont condamnés à « se retirer du marché ». Les «bonnes pratiques » sont exposées par ailleurs. Les gouvernements qui ont développé les meilleurs outils pour opérer un traitement au cas par cas sont cités en exemple (Smith, 1998). Par effet d'imitation, chacun se dote de structures institutionnelles qui assurent une gestion individualisée et individualisante des difficultés rencontrées par les viticulteurs. Cette orientation est notamment perceptible dans le dispositif Agriculteurs en difficulté (AGRIDIFF) mis en place par les autorités françaises au début des années 1990 : une ligne budgétaire est mise à la disposition du ministère de l'Agriculture, qui la répartit entre les caisses départementales de la Mutualité Sociale Agricole (MSA). Strictement conditionnée à une estimation de "viabilité » de l'exploitation, l'affectation des sommes disponibles est ensuite contrôlée par la Section Agriculteurs en Difficulté (SAD) de la Commission départementale d'orientation agricole (CDOA). Lorsque l'exploitation est considérée " non redressable », une préretraite ou un programme de réinsertion professionnelle sont simplement proposés. A titre complémentaire, la MSA crée en 2001 le Fonds de Solidarité des Crises Agricoles (FSCA) : sur cette base, elle aménage pour des exploitants touchés par une crise sectorielle un "plan d'étalement» des cotisations sociales et une "remise des majorations de retard ». En janvier 2006, la caisse audoise de la MSA s'associe à la Chambre d'Agriculture pour former un Comité de veille viticole. Animée par des travailleurs sociaux et des « conseillers en protection sociale », cette structure adresse pas moins de 1500 courriers aux familles de viticulteurs les plus modestes pour les inviter à faire connaître leurs difficultés, à s'assurer qu'ils bénéficient bien de toutes les prestations auxquelles ils ont droit, et à solliciter des conseils pour constituer leurs dossiers administratifs. 221 cas individuels 
sont ainsi examinés en 2006; 47 s'y ajoutent au premier semestre 2007. Une assistance personnalisée est notamment apportée aux viticulteurs qui souhaitent bénéficier du RMI. Sont par ailleurs relayées des offres d'emploi complémentaires transmises par l'Association pour le Développement de l'Emploi et de la Formation en Agriculture (ADEFA). 70 exploitants sont également orientés vers des formations dispensées par la Chambre d'agriculture sous les intitulés : «Vendre son vin en circuits professionnels »; « Raisonner la diversification sur son exploitation »; «Faire émerger un projet face à la crise ». Une inscription est enfin offerte à des stages organisés par le Fonds pour la Formation des Entrepreneurs du Vivant (VIVEA) ${ }^{6}$. Le document qui présente l'offre de formation met l'accent sur la responsabilité individuelle de l'exploitant et sur la nécessité de développer une stratégie entrepreneuriale avisée ${ }^{7}$. En présentant la boîte à outil "managériale » comme une clé de la réussite personnelle, ce discours confère le statut d'évidence à la concurrence avec le voisinage et transforme en incongruités l'entraide et l'échange de services, dimensions essentielles de la sociabilité en milieu rural (Vignon et Pierru, 2006).

Si les dispositifs envisagés jusqu'alors organisent une individualisation sourde et difficilement perceptible par les intéressés, ils trouvent des prolongements plus explicites et plus directement ressentis dans le traitement bancaire des situations d'endettement. En application du principe dit de "l'année blanche ", le Crédit agricole accorde à certains viticulteurs des reports de remboursements d'emprunts. Le crédit se trouve alors prolongé de douze mois avec une augmentation proportionnelle des intérêts. Cette solution est adoptée dans l'espoir d'une prochaine embellie sur le marché du vin ; faute d'une telle bouffée d'air, elle aggrave au final les difficultés rencontrées. Les viticulteurs interrogés perçoivent l'examen sourcilleux et le traitement intéressé des dossiers individuels comme une remise en cause de droits historiques. Ils estiment voir fonctionner à contresens une structure conçue à l'origine sur un mode coopératif et dans le but d'obtenir des avantages collectifs.

«On a tué la mutualisation des risques et des bénéfices. Dans les années 50, tout fonctionnait sous forme de mutualisation. C'est-à-dire que les gens y participaient et maîtrisaient leur propre devenir. Le viticulteur d'avant, c'était son propre... Maintenant il ne maîtrise plus rien. Les administrateurs, ils ont l'information, et ils se positionnent du côté de la banque contre les viticulteurs. Ils ont TOUTES les informations; ils vous en donneront PAS UNE. Ils préfèrent défendre la banque que le copain viticulteur. C'est ça qui est lamentable. C'est lamentable » (viticulteur des Corbières)

L'indignation que les uns et les autres expriment ne suffit pas à annuler les effets d'une incitation généralisée à l'appréhension individuelle des difficultés rencontrées ${ }^{8}$. Les viticulteurs qui connaissent une situation critique n'arriment pas leur souffrance à une

\footnotetext{
${ }^{6}$ VIVEA est un « fonds d'assurance formation », créé en 2001 par convention entre la Confédération Paysanne, la Coordination Rurale, la Fédération Nationale des Syndicats d'Exploitants Agricoles (FNSEA), les Jeunes Agriculteurs (JA), l'Assemblée Permanente des Chambres d'Agriculture (APCA) et la Confédération Nationale de la Mutualité, de la Coopération et du Crédit Agricole (CNMCCA).

${ }^{7}$ VIVEA, Du diagnostic à la stratégie d'entreprise. Les différentes approches et les pratiques en formation, 29 mars 2007.

${ }^{8}$ Dans les rares cas où un avantage est obtenu sur une base collective et indifférenciée, les modalités de mise en œuvre produisent un effet de fractionnement. Ainsi, pour alléger la situation financière des viticulteurs, la Direction départementale des services fiscaux de l'Aude accorde régulièrement un dégrèvement ou une exonération gracieuse de la Taxe foncière sur le non bâti (TFNB) qui devrait être prélevée sur toutes les parcelles de vigne. Or, au lieu d'être appliquée de façon automatique et centralisée, la mesure ne prend effet que si les exploitants adressent à l'administration une demande écrite individuelle.
} 
construction discursive commune. Faute d'un propos unificateur, ils peinent même à partager leurs expériences et tendent à intérioriser leur colère ${ }^{9}$.

« Le fait de ne pas avoir de revenus... C'est terrible de savoir qu'on doit des sous. C'est terrible ça (...) Bon, à un moment donné, si tu veux, c'est comme quand tu es atteint d'une maladie un peu bizarre quoi. A la fin tu en parles, mais tu sens bien que tu fais chier autour de toi. Tu es obligé de te l'encaisser quoi » (viticulteur du Minervois)

Cette configuration est le produit indirect de la politique de subventions européennes mise en œuvre dans les années 1980. Dès lors que s'impose l'idée selon laquelle les exploitants doivent développer séparément des stratégies commerciales avisées, aucune prise n'est offerte à un discours unificateur. S'ils déplorent individuellement l'adoption de mesures contraires à leurs intérêts, les viticulteurs en difficulté ne trouvent pas les supports institutionnels articulés qui pourraient relayer leurs griefs et encourager leur expression collective. Un correctif pourrait encore être trouvé si des organisations représentatives s'attachaient à objectiver un groupe au nom duquel elles prétendraient parler. Indirectement, l'intégration européenne introduit encore des obstacles sur ce plan.

\section{Un fonctionnement autarcique des structures syndicales}

Des organisations représentatives peuvent contribuer à une mobilisation collective en construisant la fiction d'un groupe unitaire dont les intérêts devraient être défendus. Un échange minimal avec les individus représentés est alors nécessaire pour entretenir un processus d'identification. L'orientation de la politique viticole européenne tend à l'inverse à favoriser un fonctionnement autarcique des structures syndicales, de sorte que les catégories délimitées par les représentants des viticulteurs ne sont pas mobilisatrices.

Très nombreux dans l'Aude, les syndicats de viticulteurs tendent à construire une opposition entre caves particulières et caves coopératives - deux catégories qui regroupent chacune des exploitants aux profils très divers (Petric \& Gouez, 2007; Lem, 1999 ; Martin, 1996). Faute d'une communication minimale avec les exploitants qu'ils prétendent représenter, les leaders syndicaux peinent cependant à imposer leurs principes de vision et de division de la viticulture.

Identifiés comme un premier ensemble, les exploitants qui travaillent dans des caves particulières se présentent volontiers comme des «vignerons indépendants ${ }^{10}$. Leurs profils sont très variables. Les propriétaires de grands domaines peuvent limiter les coûts de revient de leur production à l'hectare et écouler sans peine leur vin. Il arrive qu'ils se comportent en simples investisseurs et choisissent de ne pas résider sur place. Des caves de dimension plus modeste existent par ailleurs, le plus souvent organisées en groupements de producteurs :

\footnotetext{
${ }^{9}$ L'effet de fragmentation produit par le traitement institutionnel des difficultés rencontrées vient redoubler l'«individualisme négatif» et les stratégies de salut individuel que les populations précarisées développent lorsqu'elles ne disposent pas de ressources suffisantes pour donner un tour collectif à leurs revendications.

${ }^{10}$ La terminologie employée pour désigner les producteurs est lourde d'affrontements symboliques. La catégorie « viticulteurs » est la plus large et la plus inclusive; elle caractérise le simple fait de cultiver la vigne et de produire du raisin. La désignation «vigneron » est plus restrictive : elle identifie les exploitants qui se livrent eux-mêmes à la vinification - ce qui n'est pas le cas des coopérateurs. Un brouillage sémantique est néanmoins observé. La qualité de vigneron tend à être revendiquée pour marquer un ancrage dans une « tradition » et un attachement à développer une production artisanale, par opposition à la fabrication d'un breuvage standardisé, conçu pour être écoulé à moindres frais sur un marché mondialisé (de la même façon l'étiquette "paysan ", autrefois péjorative, peut désormais être préférée au titre d' " agriculteur »).
} 
chacun prend alors en charge la vinification de son propre raisin et parfois la mise en bouteille. La commercialisation est ensuite assurée en commun, tandis que les demandes d'aides et subventions sont centralisées. Des syndicats de crus permettent une régulation stricte. Dans ce cas, les producteurs d'un territoire délimité s'accordent sur un cahier des charges qui conditionne l'utilisation d'un label. L'hétérogénéité n'empêche pas d'afficher une prétention à l'unité : une Fédération des Vignerons indépendants de l'Aude est constituée depuis 1976 et s'emboîte elle-même dans la Fédération régionale des vignerons indépendants du Languedoc-Roussillon. Cette dernière organisation est toutefois contrôlée par les grands propriétaires. Selon son président, Jean-Marie Fabre (propriétaire du domaine de la Rochelierre à Fitou), le projet de restructuration porté par la Commission européenne « va dans le bon sens, celui de la réforme et de la compétitivité» ${ }^{11}$.

Un second ensemble regroupe les viticulteurs qui se présentent comme coopérateurs et qui se contentent de produire du raisin. La coopérative vinifie leur récolte puis vend son stock de vin à un négociant, ou le met elle-même en bouteille et tente de l'écouler dans la grande distribution. Ce mode d'organisation prête lui aussi à des positionnements très diversifiés. Des grands " groupes coopératifs vinicoles », qui disposent d'une puissante capacité commerciale, sont formés. Le groupe Val d'Orbieu, présidé par Joel Castany, est le plus emblématique d'entre eux : il rassemble 3000 viticulteurs, emploie 500 salariés, produit plus de 600000 hectolitres par an. Son chiffre d'affaires annuel dépasse 400 millions d'euros. Aux cotés des structures de ce type, des coopératives modestes fonctionnent isolément. Leurs membres subissent une baisse de revenus continue depuis plusieurs années et ne survivent qu'en ponctionnant le salaire de leur conjoint ou en exerçant une activité complémentaire. Trouvant leurs principaux débouchés dans la grande distribution, ils doivent s'aligner sur les tarifs pratiqués et imposés par les grands groupes. Les prix de revient ne leur permettent pas de rentrer dans leurs frais. Une pression économique est alors exercée qui pousse à l'absorption des caves en difficulté par les coopératives les plus "compétitives". En dépit des divergences, une Fédération départementale des caves coopératives de l'Aude prétend assurer une représentation syndicale unitaire. Mais elle est contrôlée par les grands groupes. Joël Castany en assure lui-même la vice-présidence. Le président en titre, Michel Servage, réclame une revalorisation des primes à l'arrachage pour les rendre plus incitatives. Il voit là une façon d'organiser « le plan social » d'une activité viticole qui s'est « délocalisée ». Il convient selon lui de permettre « à ceux qui veulent quitter l'activité de le faire dans la dignité $»^{12}$.

Si l'opposition entre caves particulières et caves coopératives est entretenue par les syndicats, aucune organisation alternative ne lutte pour imposer un autre principe de division : jamais un discours ne trace une frontière entre les exploitants (indépendants ou coopérateurs) qui peinent à rentrer dans leurs frais et les producteurs capables d'exporter leur vin en se positionnant sur des marchés rémunérateurs ou de le vendre à la grande distribution. De la même façon, l'opposition entre les viticulteurs qui entendent tirer parti de la réforme de l'OCM et ceux qui sont condamnés à la subir n'est portée par aucune structure syndicale.

Deux questions connexes peuvent être posées sur cette base. Pour quelle raison l'objectivation de la division entre caves particulières et caves coopératives n'emporte-t-elle pas l'adhésion totale des viticulteurs? Comment expliquer par ailleurs que cet échec ne pousse pas à la formation d'organisations concurrentes qui s'attacheraient à opérer d'autres découpages et qui prétendraient prendre en charge des groupes « mieux délimités » ? Une seule et même réponse

${ }^{11}$ «En France, c'est la commercialisation du vin qui pose problème, pas la surproduction », L'Expansion, 4 juillet 2007, p.24.

${ }^{12}$ Déclaration à l'AFP, 6 mars 2007. 
s'impose : pour asseoir la crédibilité du découpage opéré ou imposer un découpage alternatif, il conviendrait d'entretenir des échanges réguliers avec les viticulteurs. Or les représentants syndicaux tendent à évoluer dans un microcosme, sans rechercher le moindre contact avec leur base putative. Décelable aussi bien dans les syndicats de caves particulières que dans les syndicats de coopératives, cette orientation est encore le produit indirect des modes de financement européens.

Les propriétaires de caves particulières ont pris appui sur les subventions européennes pour former des groupements de producteurs; leur objectif avoué était de pratiquer une commercialisation directe en contournant le négoce local. Ils se sont ainsi engagés dans un processus dont ils ont rapidement perdu le contrôle. N'ayant plus d'emprise sur le fonctionnement du marché, les syndicats de cru semblent désormais déconnectés des circuits de décision, devenus lointains et insaisissables. Leurs dirigeants se contentent d'occuper des positions officielles et ne se soucient guère d'emporter l'adhésion des viticulteurs.

«On constate que tout le tissu de commercialisation locale qui existait dans les années 50 (Carcassonne: 12 négociants en vin; Caunes-Minervois: 12 négociants en vin - un bled de 1200 habitants ; des courtiers en vin, 3 ou 4 par village...) ce tissu, on l'a anéantit en dix ans ; on a tout foutu en l'air avec les groupements de producteurs. A partir du moment où on a cassé tout ça, le négociant ou le courtier, la coopérative ou le directeur de la cave lui disait: «j’ai pas besoin de toi, je négocie directement ». Et donc on pouvait penser qu'on maîtrisait totalement la situation. Manque de bol, on n'a fait que déplacer la prise de décision du prix du local au national ou alors maintenant mondial et on se retrouve dans un marché un peu du type boursier où vous avez des gens qui tentent des coups (...). Aujourd'hui dans votre propre groupement de producteurs, vous avez un conseil d'administration et vous avez un directeur commercial qui vous dit «ferme ta gueule, parce que la seule solution que j'ai trouvée, c'est de te vendre ton Minervois à 55 euros l'hecto. Y a pas d'autre solution! » (viticulteur du Minervois).

L'obtention régulière des aides européennes a dispensé les présidents de coopératives d'associer leurs adhérents à la prise de décision et leur a donné les moyens de contrôler sans contestation les structures syndicales. La représentation professionnelle a alors pu fonctionner en cercle fermé, sans qu'il soit besoin de rendre de comptes à la base ni de rechercher son engagement.

«Le syndicalisme est devenu élitiste parce qu'une manne européenne gérait le marché. Pédagogiquement, il n’y a jamais eu d'action menée pour amener et former des gens aux responsabilités... » (viticulteur du Minervois).

Perte d'emprise dans le cas des syndicats de caves particulières, absence de contact avec la base dans le cas des syndicats de coopératives... une pente autarcique est dans tous les cas suivie. Faute d'une organisation qui assure une mise en forme des mécontentements ${ }^{13}$, quelques viticulteurs expriment leurs griefs par des actes de violence. En 2004, un Comité Régional d'Action Viticole (CRAV) revendique le plastiquage de plusieurs agences bancaires, d'un bâtiment du Trésor public à Narbonne, de plusieurs voix ferrées ... En avril 2007, des engins incendiaires sont lancés sur des grandes surfaces ; à Narbonne, le poste électrique du magasin de discount alimentaire « Leader Price » est détruit à l'explosif et le slogan « CRAV BOUM » est peint sur les bâtiments attenants. Le 14 mai 2007, au lendemain du second tour de l'élection présidentielle, 7 viticulteurs cagoulés organisent une conférence de presse pour

\footnotetext{
${ }^{13}$ En délégitimant les mécanismes de délégation au bénéfice des procédures délibératives, la Commission empêche dans le même temps que des critères d'identification soient fournis par des partis politiques : longtemps attachés à unifier les revendications des viticulteurs, les élus socialistes de l'Aude se contentent aujourd'hui de relayer des doléances fragmentées (Roger, 2008).
} 
le compte du CRAV, dans un lieu tenu secret et en présence d'un unique journaliste qui assure la diffusion du propos sur France 3 Sud. Ils lancent alors un «ultimatum d'un mois » à Nicolas Sarkozy et appellent les viticulteurs à « passer à l'action » s'il « ne va pas dans le sens de l'intérêt viticole ».

\section{Conclusion}

S'ils incriminent la Commission européenne, les viticulteurs qui se voient précarisés ne se mobilisent pas directement contre elle. Leurs griefs ne sont pas canalisés dans un mouvement organisé ni relayés par des porte-parole clairement identifiés. Cette situation s'explique par une combinaison de différents facteurs qui trouvent leur origine dans la politique viticole européenne - sans qu'il soit possible de caractériser pour autant un plan concerté et sans que l'étouffement de la mobilisation soit un objectif explicitement formulé. Aligné sur le mécanisme de versement des subventions communautaires, le traitement institutionnel des difficultés rencontrées par les exploitants les plus modestes concourt à une appréhension individualisée et fragmentaire de la crise. Les organisations syndicales n'offrent pas les moyens de surmonter ces obstacles : la gestion des aides européennes leur permet de construire un capital institutionnel autosuffisant et les dispense de construire un groupe qu'elles prétendraient représenter. Reste à déterminer si le cas sur lequel nous concentrons notre attention présente un caractère spécifique ou si les mêmes logiques sont à l'oeuvre sous des formes plus ou moins atténuées - dans d'autres secteurs directement exposés aux politiques européennes (transports, télécommunications...).

\section{Bibliographie}

BOUMAZA Magali \& PIERRU Emmanuel (2007), «Des mouvements précaires à l'unification d'une cause 11 , Sociétés contemporaines, n65, pp. 7-25

BRESSON Maryse (2007), Sociologie de la précarité, Paris, Armand Colin

COLLOVALD Annie \& GAïTl Brigitte (1991), « Des causes qui parlent », Politix, n¹6, pp. 7-22

EBERSOLD Serge (2001), La naissance de l'inemployable, ou l'insertion aux risques de l'exclusion, Rennes, Presses universitaires de Rennes

GUILLEMIN Alain (1990), "La révolte du Midi », in COULOMB P. \& al., Les agriculteurs et la politique, Paris, Presses de la FNSP

JEGOUZO Guenahël, BRANGEON Jean-Louis, ROZE Bernard (1998), Richesse et pauvreté en agriculture, Paris, INRA-Economica

MANN Patrice (1997), « De l'ancrage cognitif à sa redéfinition. L'exemple des crises du vignoble du Midi dans la première moitié du XXe siècle "), in BOUDON Raymond \& al., Cognition et sciences sociales, Paris, PUF

MARESCA Sylvain (1983), Les dirigeants paysans, Paris, Editions de Minuit

MARTIN Jean-Philippe (1996), "Wine growers' syndicalism in the Languedoc : continuity and change ", Sociologia Ruralis, 36(3), p.331-339

PETRIC Boris \& GOUEZ Aziliz (2007), « Le vin et l'Europe : métamorphose d'une terre d'élection », Notre Europe - Etudes et Recherches, n56

PIERRU Emmanuel et VIGNON Sébastien (2006), « Déstabilisation des lieux d'intégration traditionnels et transformations de l'"entre-soi" rural. L'exemple du département de la Somme 1 , communication au colloque «Les mondes ruraux à l'épreuve des sciences sociales », MSH de Dijon, 17-19 mai

ROGER Antoine (2008), "Faire parler les viticulteurs. La construction d'un 'électorat' dans le département de l'Aude ", in ANQUETIN Virginie \& FREYERMUTH Audrey (dir), La figure de I'habitant. Sociologie politique de la "demande sociale ", Rennes, Presses Universitaires de Rennes, coll. "Res Publica ॥, 2008, p. 95-114 
SMITH Andy (1998), "L'Europe, le Midi et le vin », Pôle sud, n9, pp. 125-135

SMITH Andy, DE MAILLARD Jacques \& COSTA Olivier (2007), Vin et politique. Bordeaux, la France, la mondialisation, Paris, Presses de Sciences po 